\title{
Cooperative non-orthogonal multiple access for wireless communication networks by exploiting the EXIT chart analysis
}

\author{
Zeyad Elsaraf, Abbas Ahmed, Faheem Ahmad Khan* (il and Qasim Zeeshan Ahmed
}

\author{
${ }^{*}$ Correspondence: \\ f.khan@hud.ac.uk \\ Department of Engineering \\ and Technology, \\ School of Computing \\ and Engineering, University \\ of Huddersfield, Queensgate, \\ Huddersfield HD1 3DH, UK
}

\begin{abstract}
In the next generation of mobile communication networks, unprecedented challenges are required to be met, such as much higher data rates and spectrum efficiency, lower latency, and massive connectivity. Non-orthogonal multiple access (NOMA) has recently been proposed as a promising technology to achieve much superior spectral efficiency compared to conventional orthogonal multiple access techniques employed in present communication systems. A salient feature of NOMA is its use of successive interference cancellation (SIC) to decode users' information when multiple users are allowed to transmit in same time/frequency/code domain. In this paper, we aim to exploit an aspect of SIC, namely the availability of other users' data to realize a cooperative NOMA system. EXtrinsic information transfer (EXIT) charts are utilized to examine the performance of proposed system in terms of user fairness while employing IRregular convolutional codes (IRCC)s. The EXIT chart using IRCC evaluates the convergence analysis for the proposed system. Further, to evaluate the system performances in cooperative NOMA system, we have derived the expressions for the achievable rates which are obtained independently and utilized them in evaluating the experimental data for the proposed NOMA model.
\end{abstract}

Keywords: Extrinsic information transfer (EXIT), IRregular convolutional code (IRCC), Non-orthogonal multiple access (NOMA), Multi-user detector (MUD), Successive interference cancellation (SIC)

\section{Introduction}

The extraordinary growth of wireless connectivity in recent years has led to a number of challenging needs for the development of next-generation wireless networks (NGWNs), which are indicated by the anticipation of nearly a thousand-fold increase in data traffic, much higher spectral efficiency, lower latency and massive connectivity [1-4]. A possible solution is to utilize the non-orthogonal multiple access (NOMA) techniques. NOMA has gathered significant attraction in recent years and is considered as a promising multiple access technique for the NGWNs [5-11].

Traditionally, radio resources are exploited by multiple access schemes such as time, frequency, code, and space division which are orthogonally allocated between different author(s) and the source, provide a link to the Creative Commons licence, and indicate if changes were made. The images or other third party material in this article are included in the article's Creative Commons licence, unless indicated otherwise in a credit line to the material. If material is not included in the article's Creative Commons licence and your intended use is not permitted by statutory regulation or exceeds the permitted use, you will need to obtain permission directly from the copyright holder. To view a copy of this licence, visit http:// creativecommons.org/licenses/by/4.0/. 
users $[6,11,12]$. Thus, using the concept of orthogonality, the receiver design prevents interuser interference (IUI), thereby limiting the maximum number of available users [13]. To fulfill the diverse requirements of the NGWNs, several new multiple access techniques have been designed during the last few decades [6, 14]. Consequently, NOMA is the one which can satisfy the aforementioned demands more expediently. It is noted that NOMA can operate in combination with upcoming and existing communication technologies to fulfill the desired capacity for wireless networks, such as Massive Multiple Input Multiple Output (MIMO) [15], cooperative communications [16], cognitive radios [17], millimeter wave [18, 19], and visible light communication [20]. It has been observed that NOMA improves on the number of users supported as well as the performance of the system in various aspects.

There exist two categories of NOMA solutions: code domain NOMA (CD-NOMA) [8, 21, 22] and power domain NOMA (PD-NOMA) [7, 9]. CD-NOMA achieves the multiple access by assigning users unique spreading codes with which the signal is spread and each user can be identified $[8,22]$. The code words are sparse in design to allow for a higher number of unique codes to avoid IUI $[8,21]$. PD-NOMA operates by allowing users to share transmission resources in a way that allows for improved spectral efficiency. In this scheme, power levels are designated according to the NOMA principle $[7,9,22]$. Therefore, the user with worse channel gain is allocated higher power as compared to the user with higher channel gain. The direct result of such a system is an increased receiver complexity, while signal detection is required [6]. The complexity of the reception process is mainly due to the implementation of successive interference cancellation (SIC). The SIC process is an integral part of signal reception in a NOMA system as it allows users with lower power levels to receive their messages. SIC operates by first detecting the signal of the higher power user, by treating the other users in the signal as noise, then subtracting that signal from the received, superposed, signal, thereby extracting the signal of the low power user $[6,8]$.

One drawback of PD-NOMA is its favoring of users with worse channel conditions by providing them with nearly all the available transmission power or as much as possible, leaving users with better channel conditions with almost no power to serve their needs in some extreme cases, thereby resulting in a decrease in individual user performance $[6,8,23]$. Combining cooperative relaying with NOMA promises to alleviate many of NOMAs issues while maintaining the same level of performance. The main point to note is exploiting the properties of SIC process, namely the availability of other users' data at one user, while combining it with cooperative relaying to achieve the expected performance $[7,22]$.

In cooperative NOMA, near users with stronger channel conditions to the base station (BS) decode information for others and act as relays for far users with poor channels to the BS who are at the cell edge to improve reception reliability for them. Several cooperative NOMA schemes have been proposed in the literature from different perspectives. For example, in [24] the impact of relay selection (RS) on the performance of cooperative non-orthogonal multiple access (NOMA) is studied. A two-stage RS strategy was used to achieve the minimal outage probability among all possible relay selection schemes while realizing the maximal diversity gain. In [25], three typical structures of cooperative relaying-based NOMA, e.g., uplink, downlink and composite structure, have been 
investigated. A hybrid power allocation strategy was proposed that has lower computational complexity and reduced signaling overhead at the expense of marginal sum rate degradation.

In [26], a dual-hop cooperative relaying scheme using NOMA has been proposed where two sources communicate with their destinations in parallel over the same frequency band via a common relay. After receiving symbols transmitted in parallel by both sources with different allocated powers, the relay in this scheme forwards a superposition coded composite signal using NOMA to the destinations. The proposed protocol is demonstrated to be effective in terms of ergodic sum capacity by considering perfect and imperfect successive interference cancellation.

In this paper, we consider a cooperative PD-NOMA system where a base station serves multiple near users and a far user at the same time and frequency resources. The aim of this paper is to examine the performance of users by exploiting the concept of EXIT chart in the proposed cooperative NOMA system. In contrast to our related work [27], we use the concept of IRregular Convolutional Code (IRCC) in EXIT chart analysis. Further, the multi-user detector (MUD) is evaluated using joint alphabet algorithms in the EXIT chart analysis when deploying in single/multi-user case in the context of NOMA systems. Moreover, the throughput analysis is also carried out. To the best of our knowledge, the performance and evaluation of convergence in cooperative NOMA has not been examined before using EXIT chart by adopting IRCC. Following are the novel contributions in this paper:

- We have evaluated the achievable rate and throughput for cooperative relaying-based NOMA scheme, which is investigated by deploying more users in the system by comparing the system with/without cooperation in the NOMA model.

- We have evaluated multi-user detector (MUD) in NOMA system through EXIT chart by exploiting the IRCC.

- It is shown that joint alphabet MUD achieves higher capacity than the single user in the case of single- as well as multi-carrier scenarios.

The paper consists of the following sections: Section 2 discusses the suggested system model. In Sect. 3 the data rates are derived initially for each user while considering the IUI and the independent fading which occurs in NOMA model independently. After that the throughput analysis is conducted in NOMA model and finally the introduction of EXIT charts is carried out, where we have discussed about the diversity gains, normalization throughput and complexity evolved in MUD decoder. Furthermore, simulation results are discussed in detail in Sect. 4 and the conclusions are provided in Sect. 5.

\section{Methods and problem formulation}

The system model of the proposed NOMA system is designed and shown in Fig. 1. The data of the user are modulated by converting the information bits according to the quadrature amplitude modulation (QAM) $[8,23]$. The signal bits are then transferred to the signal spreader, where the mapped bits are multiplied by the predesigned code, which are known to the receiver. The information obtained from the signal spread, is allocated a power according to the basic principle of power allocation in NOMA mode 


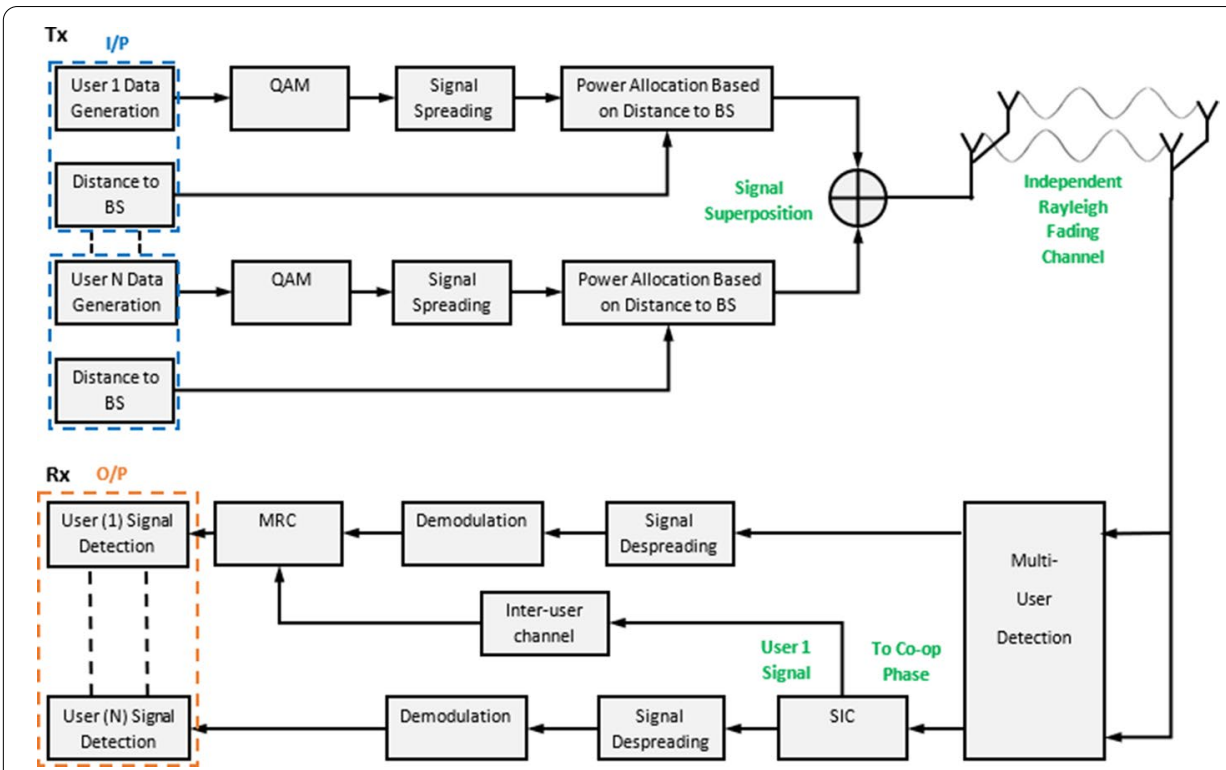

Fig. 1 Overall system model block diagram [27]

as indicated in Fig. 1. After passing through the Rayleigh fading channel, the interference signal is then received as shown in Fig. 1. On the receiver side of Fig. 1, the interference due to the channel is catered by utilizing the iteration procedure conducted in MUD where the optimal order of SIC detection relies on detecting the strongest to the weakest user. Based on this optimal SIC-detection order, any user can detect its information without substantial interference imposed on the other users whose normalized channel gain is smaller than that of this user. The signal is then despread according to the predesigned code, and demodulation is carried out at the receiver as shown in Fig. 1. So that the correct bits can be detected through the maximal ratio combining (MRC) at the receiver as shown in Fig. 1 [27], as our system model is generic and can be easily extended to multiple antennas especially for base station [28-31].

It can be observed in Fig. 2 that there are two types of users present in the cell, namely, one as the far users (FUs) and other as near users (NUs). It can be observed from Fig. 2, ' $N$ ' number of NU users are located near the BS. Two transmission phases are associated with NOMA model as indicated in Fig. 2 which are discussed below.

\subsection{Direct transmission phase (DTP)}

Figure 2 shows direct transmission phase (DTP), the first phase, which broadcasts $N$ users superimposed signal available at the BS. The superposed signal is comprised of all the messages of the users superposed together according to the NOMA concept [32]. The signal at the $n$-th user is expressed as:

$$
y_{1, n}=\sqrt{P_{1, n}} h_{1, n} x_{T}+w_{1, n},
$$

where the power allocation for the $n$th user allocating in the DTP phase is expressed as $P_{1, n} \cdot x_{T}$ represents the composite signal containing all users' messages such that $x_{T}=x_{1}+x_{2}+\cdots+x_{N}+x_{\mathrm{FU}}$ and the Additive White Gaussian Noise (AWGN) is 


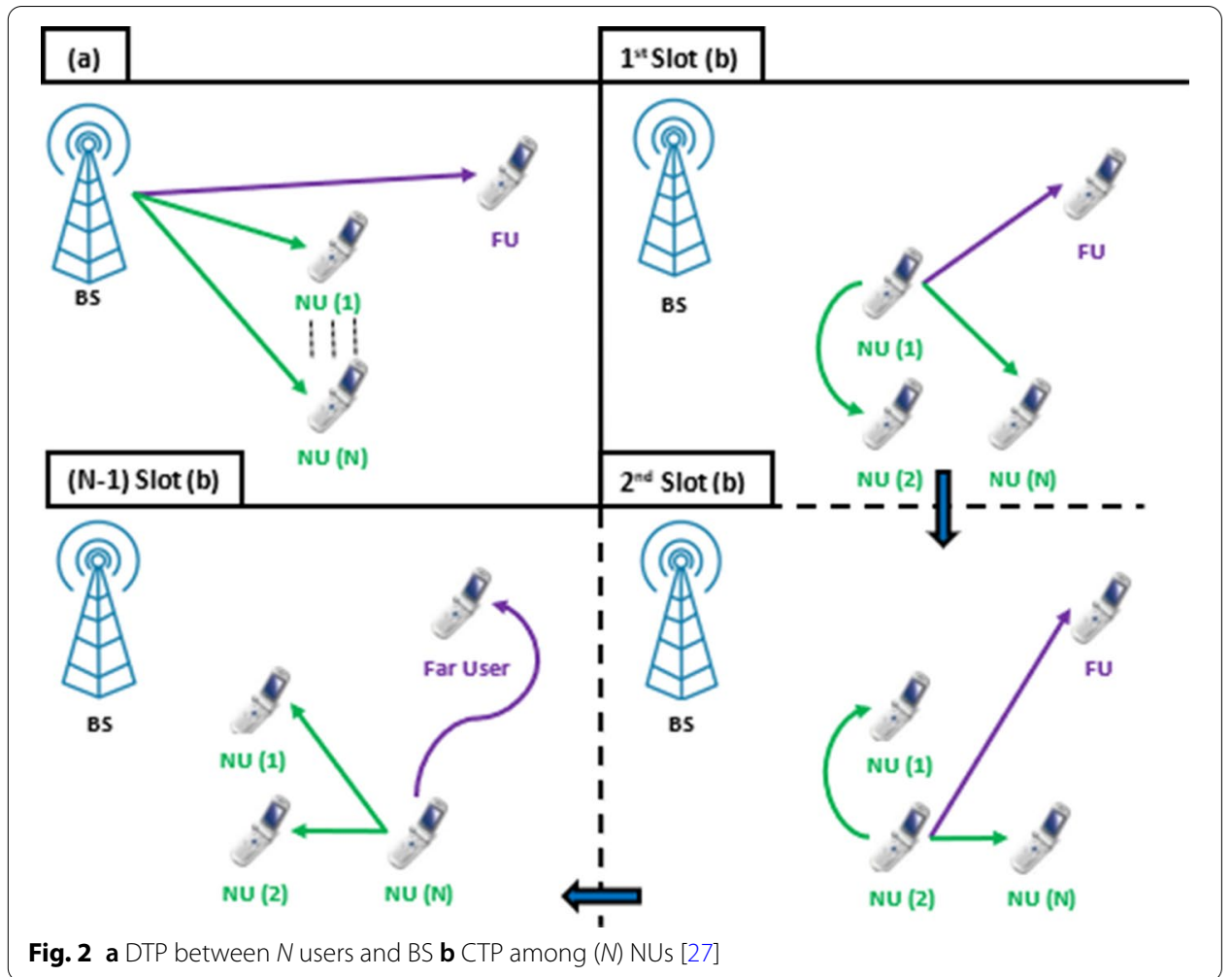

expressed as $w_{1, n}$ for each channel during the DTP phase. $h_{1, n}$ represents the independent Rayleigh fading experienced by every user channel. $d_{n}$ represents the nth user's distance away from the BS and is the basis for all power-level allocation. It is also the basis on which each user's channel condition is defined, with users closer to the BS having much better channel conditions as opposed to user much farther away from the BS.

$$
P_{1, n}=P_{t}\left(\frac{d_{n}^{2}}{\sum_{n=1}^{N+1} d_{n}^{2}}\right),
$$

where the total transmitted power available at the given BS is expressed as $P_{t}$. Furthermore, the channel conditions are expressed by [33] for NOMA model as

$$
\left|h_{1,1}\right|^{2}>\left|h_{1,2}\right|^{2} \geq \ldots \geq\left|h_{1, N}\right|^{2} \geq\left|h_{1, F U}\right|^{2}
$$

which are accordingly expressed as

$$
P_{1,1}<P_{1,2} \leq \cdots \leq P_{1, N} \leq P_{1, F U}
$$

and

$$
\sum_{n=1}^{N+1} P_{1, n}=1 .
$$

All non-cell-edge users carry out SIC to decode and isolate each user message to be exploited later during the CTP phase. 


\subsection{Cooperative transmission phase (CTP)}

Figure 2 illustrates the CTP. NU(s) utilize the information received at the end of the DTP, after applying SIC, to achieve cooperation for all users in the system [27]. During the CTP, NU(s) relay the messages for all users in one superposed signal. Each NU in the system occupies one time slot to relay the messages across the interuser channels. The CTP can be divided into $(N-1)$ time slots, with each NU occupying one time slot to relay the messages in order to avoid interuser interference (IUI). At the beginning of the CTP, user relays the messages received at the end of the DTP.

At the beginning of the CTP, $\mathrm{NU}_{1}$ broadcasts a superposed signal comprised of the data to the FU. The received signal at FU is expressed as:

$$
y_{2,2}=\sqrt{P_{2,1}} h_{2,1} x_{T-1}+w_{2,1}
$$

where $P_{2,1}$ represents the power allocation for the $\mathrm{NU}_{1}$ user allocating in the CTP phase, the superimposed signal is $x_{T-1}=x_{2}+x_{3}+\ldots+x_{N}+x_{\mathrm{FU}}, h_{2,1}$ is the Rayleigh channel coefficients between the far user and $\mathrm{NU}_{1}$ and $w_{2,1}$ is the AWGN noise. Finally, the $N$ th user will transmit to the far user. The received signal is expressed as:

$$
y_{2, N+1}=\sqrt{P_{2, N}} h_{2, N} x_{T-N}+w_{2, N},
$$

where $P_{2, N}$ represents the power allocation for the $\mathrm{NU}_{N}$ user allocating in the CTP phase, the superimposed signal is $x_{T-N}=x_{2}+x_{3}+, \cdots+x_{N-1}+x_{\mathrm{FU}}, h_{2, N}$ is the Rayleigh channel coefficients between the far and $\mathrm{NU}_{N}$ user and $w_{2, N}$ is the AWGN noise.

\section{Performance analysis}

The performance of the proposed system is measured in accordance with: the achievable rate at each user and the overall system throughput. The achievable rate of every user is in accordance with the SIC chain. The user fairness is measured according to the EXIT chart analysis.

\subsection{Achievable rate analysis}

Generally, the achievable rate is measured in bits/s/Hz. It can be observed from Fig. 2 that at the end of DTP phase, the achievable rate of the FU is evaluated as:

$$
R_{1, F U}=\log \left(1+\frac{P_{1, F U}\left|h_{1, F U}\right|^{2}}{\sum_{j=1}^{N} P_{1, j}\left|h_{1, j}\right|^{2}+N_{0_{1, F U}}}\right) .
$$

Thus, the $\mathrm{NU}_{j}$ achievable rate after DTP can be expressed as:

$$
R_{1, \mathrm{NU}_{j}}=\log \left(1+\frac{P_{1, j}\left|h_{1, j}\right|^{2}}{N_{0_{1, \mathrm{NU}}}}\right), j=1,2, \cdots, N .
$$

It can be observed from Fig. 2 that the final FU achievable rate after the CTP is expressed as: 


$$
R_{\mathrm{FU}}=\log \left(1+\frac{P_{1, F U}\left|h_{1, F U}\right|^{2}}{\sum_{j=1}^{N} P_{1, j}\left|h_{1, j}\right|^{2}+N_{0_{1, F U}}}+\sum_{j=1}^{N} \frac{P_{2, j}\left|h_{2, j}\right|^{2}}{N_{0_{j, F U}}}\right) .
$$

\subsection{System throughput analysis}

For the sake of simulations, the system throughput is defined as the additive sum of the data rates experienced at each user. This is a key performance metric and is used to measure the overall efficiency of the proposed system. The system throughput is expressed as:

$$
S=\sum_{j=1}^{N} R_{1, N U_{j}}+R_{\mathrm{FU}}
$$

\subsection{EXIT chart analysis}

EXIT charts have progressed as a tool to observe the convergence behavior of models by utilizing iterative decoding [34-36]. The signal to noise ratio (SNR) at which an extremely low bit error rate (BER) can be achieved for near-capacity performance is predicted by the use of EXIT charts without the need for Monte Carlo simulations [34, 35]. Their ultimate benefit is that they allow us to accurately estimate the above-mentioned performance metrics without requiring excessive complex Monte Carlo simulations. We utilize the implementation of EXIT charts by exchanging the information between the input/output module of MUD in NOMA system. The EXIT chart comprises of three main parts such as outer, inner curves and the stair like shape known as trajectory.

The inner and outer curves are simulated using interleavers to predict the values of the stair-shaped trajectory. When the stair-shaped trajectory matches to the inner and outer curves and moreover the open tunnel exists between the inner and outer curve, this means that the system has reached the maximum convergences that is point [1 1 1] which exist on the right corner of Fig. 8. The performances of matching the inner and outer curves depend upon the stair-shaped trajectory which is associated with the length of the interleaver which is always finite; therefore, the trajectory may not match perfectly to the inner and outer curves of the EXIT chart [34, 36, 37].

The signal mapper is used on encoded bit stream to transmit the information to the receiver, where the information is corrupted by noise. Thus, when receiving the $N$ parallel decoder chains the MUD [34,36], is used to perform log likelihood ratio (LLR) on the concerned bits. On receiving the $m$ th sub-carrier, the extrinsic LLR of the $n$th user's $z$ th bit output from the MUD is evaluated as:

$$
L_{\mathrm{MUD}, \mathrm{ex}}\left(i_{l}^{(n)}\right)=\ln \frac{\sum_{Y \in v(m, z, 0)} P\left(Z_{q} \mid Y\right)(P(Y)}{\sum_{Y \in v(m, z, 1)} P\left(Z_{q} \mid Y\right)(P(Y)}-L_{\mathrm{MUD}, \mathrm{apr}}\left(i_{l}^{(n)}\right),
$$

where $z$ of $n$th user's is obtained, having $v \in\{0,1\}$ and $n \in\left\{1,2, \ldots, \log _{2} b^{(n)}\right\}$. Hence, the a priori LLR of the $n^{\text {th }}$ user's $z$ th bit is denoted by $L_{\text {MUD,apr }}\left(i_{l}^{(n)}\right)$. Please note that in MUD scenario, the initial probability of zero's and one's bits is equiprobable; thus, the initial values in the soft registers are set to zeros as indicated in [34, 38, 39], while the $a$ 
priori symbol is denoted by $P(Y)$ which represents the multi-user probability and thus the code word is expressed as $Y=\left[Y^{(1)}, \ldots, Y^{(N)}\right]^{T}$. Furthermore, the channel probability with respect to the a priori $Y$ is expressed as $P\left(Z_{q} \mid Y\right)$ and the cost function (CF) is given as $[34,40]$.

$$
F_{C F}(Y)=P\left(Z_{q} \mid Y\right)(P(Y))=\exp \left(-\left\|Z_{q}-P H_{n} W Y\right\|^{2}\right) \times P(Y) .
$$

where $H_{n}$ is the channel states of all user present in the model and $\mathrm{W}$ is the number of transmitter and receiver antennas, both being equal. Before feeding to the channel decoder the despreading is performed on a priori LLRs, so that the extrinsic LLRs of each user are calculated by the deinterleaving which is performed on the input sequence of the user. The bit-based a posteriori LLRs are generated by channel decoder, and the data are fed to the signal spreading, where more iterations are performed among the interleavers and the MUD. After performing certain iterations ' $\mathrm{I}$ ' among the DECoding (DEC) and MUD-Despreading/Spreading, a hard decision is accomplished by the decoder.

\section{Results and discussion}

The BER performance versus SNR of the NU and the FU is depicted in Fig. 3. As expected, due to the higher power level (approximately $90 \%$ of $P_{t}$ ) as it is far away from the BS (i.e., at a distance of $10 \mathrm{~m}$ ), the FU attains a better BER compared to the NU which is assigned very less power (approximately $1 \%$ of $P_{t}$ ) based on the distance between the FU from the BS (which is $1 \mathrm{~m}$ away from BS). Data rate of every user is evaluated in Fig. 4. It is observed that at higher SNR values the FU data rate stagnates; as a result, the NU data rate exceeds it. This is because of the IUI generated by the NU onto the FU, which directly increases with channel SNR. From the results of Figs. 3 and 4, the impact of cooperative NOMA can be observed. By employing cooperative relaying, FU data rate and BER improve significantly as compared to the NOMA system without relaying.

Figure 5 shows the combined system throughput for the proposed model for both the cooperative and non-cooperative systems. The system throughput is comprised of the combined data rates of the NU and FU. It can be observed from Fig. 5, the use of

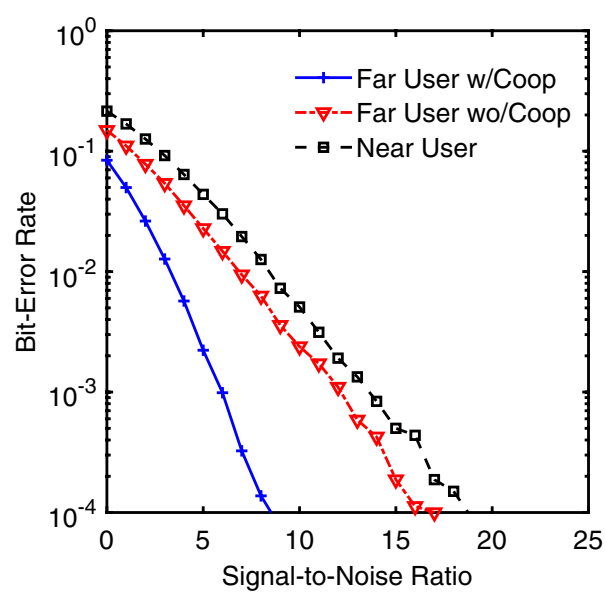

Fig. 3 BER performance comparison between near and far users 


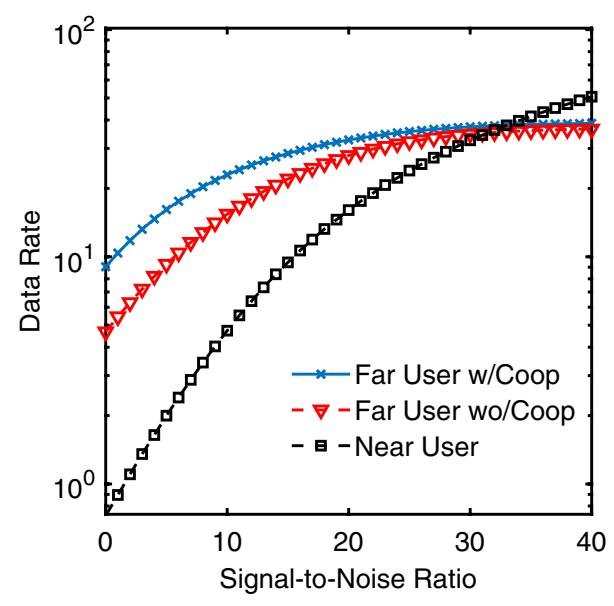

Fig. 4 Near and far user data rates

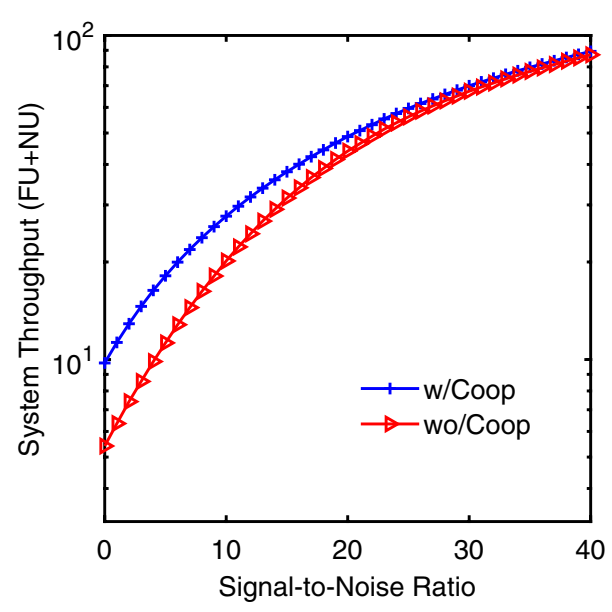

Fig. 5 System throughput for NOMA system

cooperative relaying significantly boosts the throughput of the system by a remarkable margin as the cooperative relaying result outperforms its non-cooperative counterpart. From Fig. 5 it can be seen that at $0 \mathrm{dBs}$, the system throughput for the cooperative system, starts off significantly higher than that of its non-cooperative counterpart and remains this way until $40 \mathrm{dBs}$. The difference in the performance decreases as the SNR value for the channel increases and the system approaches its capacity limit. The performance, however, reaches a point of stagnation at around $40 \mathrm{dBs}$ in accordance with Shannon's capacity law $[34,40]$.

Figure 6 presents the EXIT chart analysis, where the inner decoder utilizes the mutual information (MI) of the MUD and the outer decoder exploits the despreading/channel decoding information. The only additional computation in the MUD is to calculate the joint alphabet probability, which is carried out in an offline mode at the user's and at the BS's receiver. This is done by utilizing the fair selection of the codewords in parallel with the predetermined values of the interleavers of our system. To elaborate a little further the MUD decoder generates the bit-based a posteriori LLRs for their code words, 


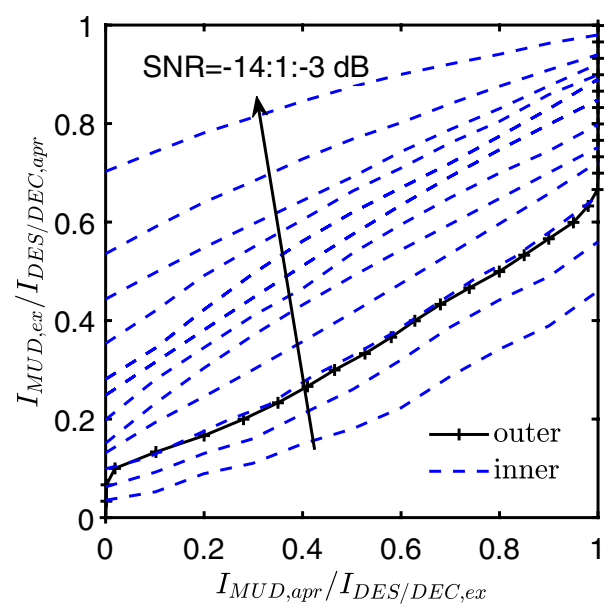

Fig. 6 EXIT chart of a NOMA system, where the outer decoder operates with RSC code having SF $=2$ when the inner decoder for various values of SNR is used in MUD model

which is later fed to the DS spreading, then to the interleavers and, finally, to the MUD for more iterations. Thus, for the joint spreading, the coding rate is calculated as $R / \mathrm{SF}$, which in our case is 0.25 . This is linked with the Spreading Factor $(\mathrm{SF}=2)$ and $R=1 / 2$. From Fig. 6 it can be concluded that the minimum required SNR is equal to $-12 \mathrm{~dB}$ for MUD to achieve a desired output. Thus, by using the concept of recursive systematic convolutional (RSC) code, along with a repetition $\mathrm{SF}=2$, the minimum SNR can achieve error-free transmission, such that suitable number of iterations are performed between the MUD $D_{D E S}$ and MUD $D_{D E C}$. The loss occurred by the MUD is pertaining to the fact that initial MI for $I_{\mathrm{MUD} \text {,apr }}=0$, as the probability of zero's and one's are the same at the beginning. Therefore, higher SNR is required for generating the open tunnel between the inner and outer curves. It can be observed that when outer code is combined with the MUD model it reaches to the $I_{\mathrm{DES} / \mathrm{DEC}, \mathrm{ex}}=1$ line at relatively low $I_{\mathrm{DES} / \mathrm{DEC} \text {,apr values }}$ as indicated in Fig. 6

From Fig. 7, it can be inferred that the proposed NOMA model attains user fairness as inner/outer curves reaches to the [1 1 ] point without crossover at any given point. Figure 7 illustrates that there is a high inclination in the inner curve as compared to the outer curve when deployed in MUD model. Moreover, the decoding trajectory depends on the iteration among the MUD and the despreader of our proposed system. Furthermore, at an $\mathrm{SNR}=3 d B$ the open EXIT tunnel exists between the inner and outer curves by performing 1000 iterations in $M U D_{D E S} / D E C$, where the inner and outer curves meet at $I_{\mathrm{MUD}, \mathrm{ex}}=0.89$ and $I_{\mathrm{DES} / \mathrm{DEC}, \mathrm{ex}}=1$ as shown in Fig. 7 .

Finally, Fig. 8 is generated to calculate the normalized throughput of a single-carrier and multi-user carrier model when number of users are $U=1$ and $U=2$, deployed in non-dispersive Rayleigh channels. Figure 8 depicts that at the same instant, both the multi-user and single carrier user obtain better throughput gain, when deployed in the decoding model. Moreover, in the single carrier system, the throughput gain is higher as compared to the multi-user model, which illustrates that more transmitting antennas combinations are utilized in the single carrier system. In single carrier system 10 transmitting antennas are used, whereas in multi-user model 2 transmitting antennas 


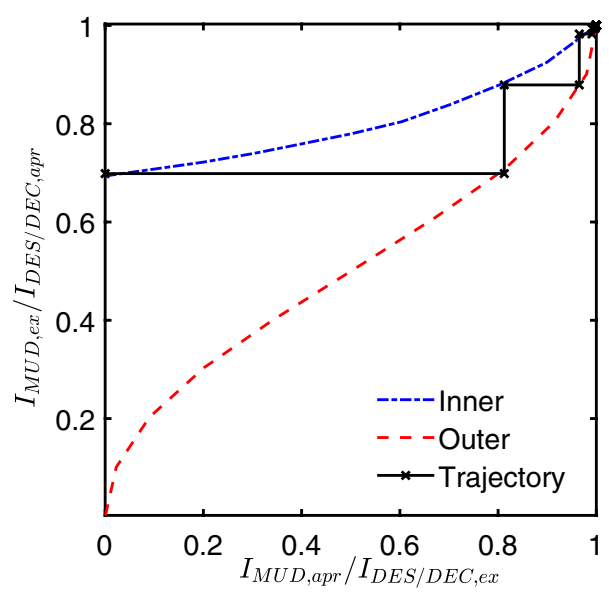

Fig. 7 EXIT chart analysis for NOMA system

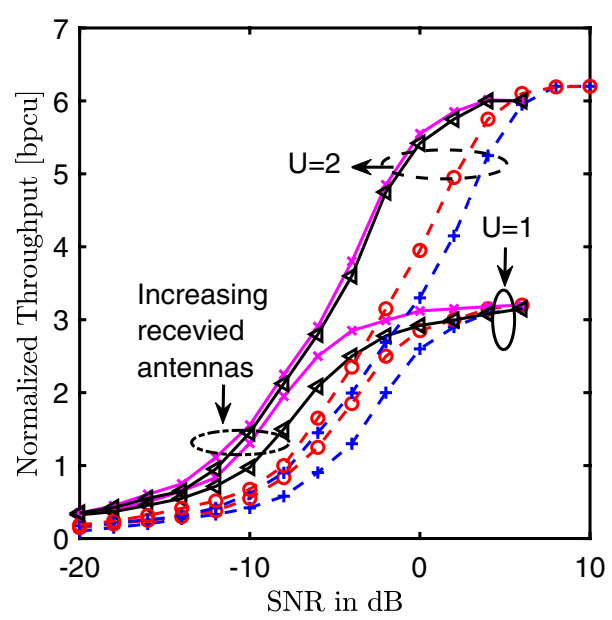

Fig. 8 Normalized throughput analysis for MUD model

are utilized. This result reveals that using more antenna array combination in single user decreases the correlation between the code word which is utilized in decoding the message, hence allowing fair selection of antenna array combination which is uniformly distributed over the single-user model.

When employing cooperative communications, reliability and security of the system get impacted. A number of authors [41-45] have addressed this issue, and the readers are encouraged to review these papers and references in them on security of cooperative NOMA.

\section{Conclusion}

In this paper, cooperative relaying was shown to achieve significantly improved performance for the proposed NOMA system. User fairness was also shown to be at nearoptimal performance through our utilization of EXIT charts. This is due to the inner and outer curves of the EXIT charts converging toward unity gain without any intersection 
at any point beforehand. Furthermore, our MUD approach with the implementation of IRCC coding was illustrated through the use of EXIT charts and was shown to offer a flexibility in the coding rate which results in an increase in overall throughput efficiency; thereby, a design methodology for combining SISO MUD with IRCC is presented. The benefits of embracing cooperative relaying in NOMA system are made clear. As a result, the proposed NOMA system model can be applied in wireless networks to strike a balance between user fairness and user data rates. Expanding the proposed cooperative NOMA system to function in a MIMO scenario while maintaining a practical level of user fairness is a promising future direction of our proposed research.

\begin{abstract}
Abbreviations
1G: First generation; 4G: Fourth generation; 5G: Fifth generation; I MuD: Iteration in multi-user detector; IDEC/IDES: Iteration in despreading/spreading module; AMA: Amenable multiple access; AWGN: Additive White Gaussian noise; BER: Bit error rate; BDM: Bit division multiplexing; BS: Base station; CDMA: Code division multiple access; CD-NOMA: Code domain NOMA; CF: Cost function; CTP: Cooperative transmission phase; DEC: Decoding; DES: Decoding iteration through despreading/spreading; DTP: Direct transmission phase; EXIT: EXtrinsic information transfer; FU: Far user; GB: Guard band; IDMA: Interleave division multiple access; loT: Internet of things; IRCC: IRregular convolutional code; ITU: International telecommunications union; IUI: Interuser interference; LDS-CDMA: Low density spreading-code division multiple access; LDS-OFDMA: Low density spreading-orthogonal frequency division multiple access; LLR: Log likelihood ratio; LoS: Line of sight; MAP: Maximum a posteriori; MI: Mutual information; MIMO: Multiple input multiple output; MUD: Multi-user detector; MUSA: Multi-user shared access; MRC: Maximal ratio combining; NGWN: Next-generation wireless network; NOMA: Non-orthogonal multiple access; NU: Near user; OFDMA: Orthogonal frequency division multiple access; OMA: Orthogonal multiple access; PDMA: Pattern division multiple access; PD-NOMA: Power domain NOMA; QAM: Quadrature amplitude modulation; RSC: Recursive systematic convolutional; SCMA: Sparse code multiple access; SDMA: Spatial division multiple access; SIC: Successive interference cancellation; SICAMA: SIC-amenable multiple access; SISO: Single input single output; SF: Spreading factor; SNR: Signal to noise ratio; UE: User equipment; URC: Unity rate codes.
\end{abstract}

\title{
Acknowledgements
}

Not applicable.

\section{Author's contributions}

The research presented in this paper is mainly formulated, analyzed, simulated and authored by ZE as a part of his PhD work. AA contributed to simulations, analysis and in revising the manuscript. FK and QA supervised the research work, gave technical inputs and reviewed the manuscript. All authors read and approved the final manuscript.

\section{Funding}

None.

Availability of data and materials

The paper is self-contained. Simulation description and parameters are provided in details in Sect. 4.

\section{Declarations}

Ethics approval and consent to participate

Not applicable.

\section{Competing interests}

The authors declare that they have no competing interests.

\section{Consent for publication}

Not applicable.

Received: 16 November 2020 Accepted: 24 March 2021

Published online: 07 April 2021

\section{References}

1. C.B. Papadias, T. Ratnarajah, D.T.M. Slock (eds.), Spectrum Sharing: The Next Frontier in Wireless Networks (Wiley, New York, 2020)

2. G.K. Papageorgiou, K. Voulgaris, K. Ntougias, D.K. Ntaikos, M.M. Butt, C. Galiotto, N. Marchetti, V. Frascolla, H. Annouar, A. Gomes, A.J. Morgado, M. Pesavento, T. Ratnarajah, K. Gopala, F. Kaltenberger, D.T.M. Slock, F.A. Khan, C.B. Papadias, Advanced dynamic spectrum 5G mobile networks employing licensed shared access. IEEE Commun. Mag. 58(7), $21-27(2020)$ 
3. A. Gupta, R.K. Jha, A survey of $5 \mathrm{G}$ network: architecture and emerging technologies. IEEE Access 3, 1206-1232 (2015)

4. M. Agiwal, A. Roy, N. Saxena, Next generation $5 G$ wireless networks: a comprehensive survey. IEEE Commun. Surv. Tutorials 18(3), 1617-1655 (2016)

5. L. Dai, B. Wang, Y. Yuan, S. Han, I. Chih-lin, Z. Wang, Non-orthogonal multiple access for 5G: solutions, challenges, opportunities, and future research trends. IEEE Commun. Mag. 53(9), 74-81 (2015)

6. S.M.R. Islam, N. Avazov, O.A. Dobre, K. Kwak, Power-domain non-orthogonal multiple access (NOMA) in 5G systems: potentials and challenges. IEEE Commun. Surveys Tutor. 19(2), 721-742 (2017)

7. M. Vaezi, R. Schober, Z. Ding, H.V. Poor, Non-orthogonal multiple access: common myths and critical questions. IEEE Wirel. Commun. 26(5), 174-180 (2019)

8. L. Dai, B. Wang, Z. Ding, Z. Wang, S. Chen, L. Hanzo, A survey of non-orthogonal multiple access for 5G. IEEE Commun. Surv. Tutorials 20(3), 2294-2323 (2018)

9. Z. Ding, Y. Liu, J. Choi, Q. Sun, M. Elkashlan, I. Chih-Lin, H.V. Poor, Application of non-orthogonal multiple access in LTE and 5G networks. IEEE Commun. Mag. 55(2), 185-191 (2017)

10. Y. Liu, Z. Qin, M. Elkashlan, Z. Ding, A. Nallanathan, L. Hanzo, Nonorthogonal multiple access for $5 G$ and beyond. Proc. IEEE 105(12), 2347-2381 (2017)

11. Q.Z. Ahmed, K.-H. Park, M.-S. Alouini, S. Aïssa, Linear transceiver design for nonorthogonal amplify-and-forward protocol using a bit error rate criterion. IEEE Trans. Wirel. Commun. 13(4), 1844-1853 (2014)

12. M.S. Ali, H. Tabassum, E. Hossain, Dynamic user clustering and power allocation for uplink and downlink nonorthogonal multiple access (NOMA) systems. IEEE Access 4, 6325-6343 (2016)

13. Z. Ding, X. Lei, G.K. Karagiannidis, R. Schober, J. Yuan, V.K. Bhargava, A survey on non-orthogonal multiple access for 5G networks: research challenges and future trends. IEEE J. Sel. Areas Commun. 35(10), 2181-2195 (2017)

14. B. Makki, K. Chitti, A. Behravan, M. Alouini, A survey of NOMA: current status and open research challenges. IEEE Open J. Commun. Soc. 1, 179-189 (2020)

15. A.S. de Sena, F.R.M. Lima, D.B. da Costa, Z. Ding, P.H.J. Nardelli, U.S. Dias, C.B. Papadias, Massive MIMO-NOMA networks with imperfect SIC: design and fairness enhancement. IEEE Trans. Wirel. Commun. 19(9), 6100-6115 (2020)

16. F.A. Khan, M.C. Fillippou, M. Sellathurai, Cooperative communication techniques for spectrum sharing, in Spectrum Sharing: The Next Frontier in Wireless Networks. ed. by C.B. Papadias, T. Ratnarajah, D.T.M. Slock (Wiley, New York, 2020), pp. $147-167$

17. F.A. Khan, C. Masouros, T. Ratnarajah, Interference-driven linear precoding in multiuser MISO downlink cognitive radio network. IEEE Trans. Veh. Technol. 61(6), 2531-2543 (2012)

18. L. Dai, B. Wang, M. Peng, S. Chen, Hybrid precoding-based millimeter-wave massive MIMO-NOMA with simultaneous wireless information and power transfer. IEEE J. Sel. Areas Commun. 37(1), 131-141 (2019)

19. O. Alluhaibi, Q.Z. Ahmed, E. Kampert, M.D. Higgins, J. Wang, Revisiting the energy-efficient hybrid DA precoding and combining design for mm-wave systems. IEEE Trans. Green Commun. Netw. 4(2), 340-354 (2020)

20. M. Vaezi, G.A. Aruma Baduge, Y. Liu, A. Arafa, F. Fang, Z. Ding, Interplay between NOMA and other emerging technologies: a survey. IEEE Trans. Cogn. Commun. Netw. 5(4), 900-919 (2019)

21. M. Moltafet, N.M. Yamchi, M.R. Javan, P. Azmi, Comparison study between PD-NOMA and SCMA. IEEE Trans. Veh. Technol. 67(2), 1830-1834 (2018)

22. Z. Elsaraf, F. Khan, Q. Ahmed, Performance analysis of code- domain NOMA in 5G communication systems, in 2019 Proceedings of the Emerging Technologies Conference, Huddersfield, UK, 2019

23. X. Dai, S. Chen, S. Sun, S. Kang, Y. Wang, Z. Shen, J. Xu, Successive interference cancelation amenable multiple access (SAMA) for future wireless communications, in 2014 IEEE International Conference on Communication Systems, pp. 222-226 (2014)

24. Z. Yang, Z. Ding, Y. Wu, P. Fan, Novel relay selection strategies for cooperative noma. IEEE Trans. Veh. Technol. 66(11), 10114-10123 (2017)

25. D. Wan, M. Wen, F. Ji, H. Yu, F. Chen, Non-orthogonal multiple access for cooperative communications: challenges, opportunities, and trends. IEEE Wirel. Commun. 25(2), 109-117 (2018)

26. M.F. Kader, M.B. Shahab, S.Y. Shin, Exploiting non-orthogonal multiple access in cooperative relay sharing. IEEE Commun. Lett. 21(5), 1159-1162 (2017)

27. Z. Elsaraf, A. Ahmed, F.A. Khan, Q.Z. Ahmed, EXIT chart analysis of cooperative non-orthogonal multiple access for next generation wireless communication systems, in 2020 European Conference on Networks and Communications (EuCNC), pp. 281-285 (2020)

28. Y. Li, M. Jiang, Q. Zhang, Q. Li, J. Qin, Cooperative non-orthogonal multiple access in multiple-input-multiple-output channels. IEEE Trans. Wirel. Commun. 17(3), 2068-2079 (2018)

29. N. Nonaka, Y. Kishiyama, K. Higuchi, Non-orthogonal multiple access using intra-beam superposition coding and sic in base station cooperative mimo cellular downlink, in 2014 IEEE 80th Vehicular Technology Conference (VTC2014-Fall), pp. 1-5 (2014)

30. M.B. Uddin, M.F. Kader, S.Y. Shin, Cooperative relaying using mimo noma, in 20184 th International Conference on Wireless and Telematics (ICWT), pp. 1-6 (2018)

31. O. Maraqa, A.S. Rajasekaran, S. Al-Ahmadi, H. Yanikomeroglu, S.M. Sait, A survey of rate-optimal power domain noma with enabling technologies of future wireless networks. IEEE Commun. Surv. Tutorials 22(4), 2192-2235 (2020)

32. R. Razavi, R. Hoshyar, M.A. Imran, Y. Wang, Information theoretic analysis of LDS scheme. IEEE Commun. Lett. 15(8), 798-800 (2011)

33. Z. Yuan, G. Yu, W. Li, Y. Yuan, X. Wang, J. Xu, Multi-user shared access for internet of things, in 2016 IEEE 83 rd Vehicular Technology Conference (VTC Spring) (2016)

34. M. El-Hajjar, L. Hanzo, EXIT charts for system design and analysis. IEEE Commun. Surv. Tutorials 16(1), 127-153 (2014)

35. S. ten Brink, Designing iterative decoding schemes with the extrinsic information transfer chart. AEU Int. J. Electron. Commun. 54(6), 389-398 (2000)

36. A. Ahmed, P. Botsinis, S. Won, L. Yang, L. Hanzo, Primitive polynomials for iterative recursive soft sequential acquisition of concatenated sequences. IEEE Access 7, 13882-13900 (2019) 
37. A. Ahmed, Iterative initial synchronization in wireless communications. PhD thesis, University of Southampton (2019)

38. A. Ahmed, P. Botsinis, S. Won, L. Yang, L. Hanzo, EXIT chart aided convergence analysis of recursive soft m-sequence initial acquisition in nakagami-m fading channels. IEEE Trans. Veh. Technol. 67(5), 4655-4660 (2018)

39. L. Hanzo, T.H. Liew, B.L. Yeap, R.Y.S. Tee, S.X. Ng, Turbocoded Adaptive Modulation versus Spacetime Trellis Codes for Transmission over Dispersive Channels, pp. 233-252 (2011)

40. M.Y. Alias, S. Chen, L. Hanzo, Multiple-antenna-aided OFDM employing genetic-algorithm-assisted minimum bit error rate multiuser detection. IEEE Trans. Veh. Technol. 54(5), 1713-1721 (2005)

41. B. He, A. Liu, N. Yang, V.K.N. Lau, On the design of secure non-orthogonal multiple access systems. IEEE J. Sel. Areas Commun. 35(10), 2196-2206 (2017)

42. Y.Wu, A. Khisti, C. Xiao, G. Caire, K. Wong, X. Gao, A survey of physical layer security techniques for $5 \mathrm{~g}$ wireless networks and challenges ahead. IEEE J. Sel. Areas Commun. 36(4), 679-695 (2018)

43. H. Lei, J. Zhang, K. Park, P. Xu, I.S. Ansari, G. Pan, B. Alomair, M. Alouini, On secure noma systems with transmit antenna selection schemes. IEEE Access 5, 17450-17464 (2017)

44. Y. Alsaba, C.Y. Leow, S.K. AbdulRahim, Null-steering beamforming for enhancing the physical layer security of nonorthogonal multiple access system. IEEE Access 7, 11397-11409 (2019)

45. Y. Feng, S. Yan, C. Liu, Z. Yang, N. Yang, Two-stage relay selection for enhancing physical layer security in non-orthogonal multiple access. IEEE Trans. Inf. Forensics Secur. 14(6), 1670-1683 (2019)

\section{Publisher's Note}

Springer Nature remains neutral with regard to jurisdictional claims in published maps and institutional affiliations.

\section{Submit your manuscript to a SpringerOpen ${ }^{\circ}$ journal and benefit from:}

- Convenient online submission

- Rigorous peer review

- Open access: articles freely available online

- High visibility within the field

- Retaining the copyright to your article

Submit your next manuscript at $>$ springeropen.com 